\title{
Linear signaling in the Toll-Dorsal pathway of Drosophila: activated Pelle kinase specifies all threshold outputs of gene expression while the bHLH protein Twist specifies a subset
}

\author{
Angelike Stathopoulos and Michael Levine* \\ Department of Molecular and Cell Biology, Division of Genetics and Development, 401 Barker Hall, University of California, \\ Berkeley, CA 94720, USA \\ *Author for correspondence (e-mail: mlevine@uclink4.berkeley.edu) \\ Accepted 30 April 2002
}

\section{SUMMARY}

Differential activation of the Toll receptor leads to the formation of a broad Dorsal nuclear gradient that specifies at least three patterning thresholds of gene activity along the dorsoventral axis of precellular embryos. We investigate the activities of the Pelle kinase and Twist basic helix-loop-helix (bHLH) transcription factor in transducing Toll signaling. Pelle functions downstream of Toll to release Dorsal from the Cactus inhibitor. Twist is an immediate-early gene that is activated upon entry of Dorsal into nuclei. Transgenes misexpressing Pelle and Twist were introduced into different mutant backgrounds and the patterning activities were visualized using various target genes that respond to different thresholds of Toll-Dorsal

\section{INTRODUCTION}

Dorsal is distributed in a broad concentration gradient in the precellular Drosophila embryo (reviewed by Belvin and Anderson, 1996; Rusch and Levine, 1996). It establishes dorsoventral patterning by regulating a number of target genes in a concentration-dependent fashion. At least five thresholds of gene activity have been described (Huang et al., 1997). Peak levels of Dorsal are required for the activation of the snail gene in the presumptive mesoderm in ventral regions of the early embryo (Ip et al., 1992a), whereas progressively lower levels of Dorsal activate single-minded ( $\mathrm{sim})$, ventral nervous system defective (vnd), rhomboid (rho) and short gastrulation (sog) in the neurogenic ectoderm (Kasai et al., 1998; Ip et al., 1992b; von Ohlen and Doe, 2000; Francois et al., 1994). These differential patterns of gene activity subdivide the embryo into three primary tissues: mesoderm, neurogenic ectoderm and dorsal ectoderm.

The restricted activation of the Toll receptor results from the localized processing of the Spätzle (Spz) ligand in ventral regions of the precellular embryo (Mizuguchi et al., 1998; Sen et al., 1998). Toll signaling is transduced by the Pelle kinase, which causes the direct or indirect phosphorylation and degradation of Cactus, so that Dorsal is released from the cytoplasm and enters nuclei (Grosshans et al., 1994; Galindo signaling. These studies suggest that an anteroposterior gradient of Pelle kinase activity is sufficient to generate all known Toll-Dorsal patterning thresholds and that Twist can function as a gradient morphogen to establish at least two distinct dorsoventral patterning thresholds. We discuss how the Dorsal gradient system can be modified during metazoan evolution and conclude that Dorsal-Twist interactions are distinct from the interplay between Bicoid and Hunchback, which pattern the anteroposterior axis.

Key words: Dorsoventral patterning, Drosophila embryo, Dorsal, Pelle, Twist, Gradient thresholds et al., 1995; Towb et al., 1998). A constitutively activated form of Pelle was created by fusing the Pelle kinase catalytic domain to the signal peptide, the extracellular domain and the transmembrane peptide of a constitutively activated form of the Torso (Tor) receptor, Tor ${ }^{4021}$ (Y327C) (Sprenger and NüssleinVolhard, 1992). The resulting 'Pelle-Tor ${ }^{4021}$ ' chimera caused a dominant ventralized phenotype when its RNA was injected throughout the embryo in a manner independent of both Toll receptor and Tube adapter protein (Grosshans et al., 1994; Galindo et al., 1995). This is consistent with the idea that activation of Pelle leads to efficient degradation of Cactus, so that Dorsal can enter nuclei in both dorsal and ventral regions of the mutant embryo. However, mutant phenotypes were assessed predominantly on the basis of cuticular defects. Consequently, it is not known whether physiological levels of activated Pelle kinase are sufficient for the activation of type I target genes, such as twist and snail, which depend on peak concentrations of the Dorsal gradient (Huang et al., 1997). It is conceivable that the Toll-Dorsal signaling pathway is branched. For example, activation of the Toll receptor might induce Pelle and an additional kinase, which together activate type I target genes (e.g. Karin, 1999).

twist is one of the first genes activated by the Dorsal gradient (Thisse et al., 1991; Jiang et al., 1991). It encodes a bHLH regulatory protein implicated in mesoderm differentiation in a 
broad spectrum of animals (Reuter and Leptin, 1994; Baylies and Bate, 1996; Harfe et al., 1998). This conservation of Twist contrasts with the apparently specific use of Dorsal as a dorsoventral determinant in insect embryos (Chen et al., 2000). Thus far, Rel-containing transcription factors have not been implicated in the dorsoventral patterning of vertebrate embryos, even though fly and frog embryos employ many common signaling components such as transforming growth factor $\beta$ (TGF $\beta$ ) and Chordin/Sog inhibitors (Ferguson, 1996).

Linearity in the Toll-Dorsal signaling pathway was analyzed by creating ectopic anterior-posterior gradients of Twist and activated Pelle kinases (Pelle-Tor ${ }^{4021}$ and Pelle-Tor). Twist- and Pelle-coding sequences were attached to the bicoid (bcd) 3'UTR and expressed in the maternal germline using the hsp83 promoter (Huang et al., 1997). The Pelle-Tor ${ }^{4021}$ fusion gene is sufficient to establish sequential patterns of snail, sim, vnd and sog expression across the anteroposterior axis of transgenic embryos. These results suggest that the levels of activated Pelle kinase determine dorsoventral patterning thresholds, and argue against branching in the Toll signaling pathway. The twist transgene was able to activate snail, sim and vnd expression in mutant embryos containing low, uniform levels of the Dorsal protein. However, gene expression supported by Twist in the absence of a Dorsal gradient is erratic, in that expression patterns are out of order or are incorrect. These observations contrast with the recent demonstration that most Bicoid gradient thresholds are generated also by Hunchback, an immediate target of the Bicoid activator. As Twist largely fails to compensate for the loss of the Dorsal gradient, we conclude that Dorsal and Twist function in a highly interdependent manner to specify the mesoderm and ventral regions of the neurogenic ectoderm.

\section{MATERIALS AND METHODS}

\section{Plasmid constructions, P-element transformation and in situ hybridization}

The construction of Pelle-Tor ${ }^{4021}$ and Pelle-Tor fusion proteins in pSELECT vector (Promega) have been described previously (Galindo et al., 1995). The chimeric proteins contain the extracellular and transmembrane domains of Tor and Tor ${ }^{4021}$ residues $1-455$ fused to residues 163-501 of Pelle. To generate anteroposterior Pelle-Tor and Pelle-Tor ${ }^{4021}$ gradients, we placed these fusions under the control of the hsp83 promoter and regulation by the bcd 3'UTR as follows. Blunted-NcoI/SacI fragments containing the Pelle-Tor- and PelleTor ${ }^{4021}$-coding sequences were cloned into the blunted-HindIII/SacI digested hsp83/pUC19 (Huang et al., 1997). A three-way ligation of a $K p n I / b l u n t e d-S a c I$ digested fragment containing hsp83-Pelle-Tor or hsp83-Pelle-Tor ${ }^{4021}$, blunted-SacII/XbaI fragment containing the $b c d$ 3'UTR, and KpnI/XbaI-digested Casper AUG $\beta$-gal P-element transformation vector (Huang et al., 1997) generated the Pelle-Tor ${ }^{4021}$ and Pelle-Tor P-element transformation vectors used in this study. A blunted HindIII/HincII fragment containing the entire twist open reading frame $(\mathrm{ORF})$ and a $5^{\prime} \beta$-globin leader was isolated from the Twist/pNB40 plasmid (kindly provided by Maria Leptin) and cloned into the EcoRV site of pBSK+-KpnII, creating Twist-KpnII/SK+. pBSK+-KpnII is pBSK+ (Stratagene), which was modified to contain an additional $K p n I$ site in place of $S a c I$. A $K p n I$ fragment containing the twist ORF from twist-KpnII/SK+ was inserted into the KpnI site of the pCASPER-hsp83-KpnI-bcd3'UTR vector (a modified form of pCAS-AUGßgal obtained from Hailan Zhang). twist was thereby placed under the control of the hsp83 maternal promoter and bcd3'UTR localization sequence to generate P-element transformation vector $\mathrm{P}$ (Twist-bcd). The construction of $\mathrm{P}\left(\mathrm{bcdToll}{ }^{10 \mathrm{~b}} \mathrm{bcd}\right)$ and $\mathrm{P}\left(\mathrm{hspToll}{ }^{10 \mathrm{~b}} \mathrm{bcd}\right)$ P-element transformation vectors have been previously described (Huang et al., 1997) which places the Toll ${ }^{10 b}$ sequence under control of the bcd 3'UTR localization sequence and the $b c d$ and $h s p 83$ promoters, respectively. The $b c d$ promoter mediates maternal expression at levels lower than the hsp83 maternal promoter. P-element mediated transformation and in situ hybridization using digoxigenin-labeled RNA probes were performed as described previously (Jiang et al., 1991).

\section{Genetic crosses}

All mutant fly stocks were obtained from the Bloomington Stock Center (Indiana) and wild-type embryos correspond to the $y w$ fly stock, unless otherwise noted. The ectopic Toll ${ }^{10 b}$, Pelle-Tor ${ }^{4021}$, Pelle-Tor and Twist-bcd anteroposterior Dorsal gradients were examined in the absence of the endogenous dorsoventral Dorsal gradient by introducing the Toll ${ }^{10 \mathrm{~b}}$, Pelle-Tor ${ }^{4021}$, Pelle-Tor and Twistbcd transgenes into embryos homozygous for a null mutation in gastrulation defective ( $g d 7$ ) (Konrad et al., 1988). Male flies from several independent lines carrying these P-element transposons were individually crossed to $g d 7 / \mathrm{FM} 3$ females. $g d^{7}$; (P-element)/+ males were crossed to $g d^{7} / \mathrm{FM} 3$ females and embryos from $g d^{7} / g d^{7}$; (P-element)/+ females were collected for analysis by in situ hybridization.

The ectopic Toll ${ }^{10 \mathrm{~b}}$ anteroposterior Dorsal gradient was examined in the absence of Twist by crossing the $\mathrm{P}\left(\mathrm{hspToll}{ }^{10 \mathrm{~b}} \mathrm{bcd}\right)$ transgene into a twist-/twist- mutant background. $\mathrm{Sp} / \mathrm{CyO} ; \mathrm{P}\left(\mathrm{hspToll}{ }^{10 \mathrm{~b}} \mathrm{bcd}\right) /+$ males were crossed to $c n t w i^{I} b w / \mathrm{CyO}$ females obtained from Bloomington stock center. Virgin females of the genotype $c n t w i^{l}$ $b w / \mathrm{CyO} ; \mathrm{P}\left(\mathrm{hspToll}{ }^{10 \mathrm{~b}} \mathrm{bcd}\right)$ were mated to $c n t w i^{1} b w / \mathrm{CyO}$ males. twist-/twist- mutant embryos represented one fourth of the embryos examined. Similar crosses were done to introduce the P(Twist-bcd) transgene into a snail mutant background $\left(y w\right.$; snail ${ }^{I I G 05} / \mathrm{CyO} \mathrm{ftz}-$ lacZ; kindly provided by Tony Ip).

The ectopic Twist anteroposterior gradient was examined in a background of low uniform Dorsal levels by misexpressing the twist$b c d$ transgene in embryos homozygous for a partially activating mutation of Toll (Schneider et al., 1991). Male flies from several independent lines in which the $\mathrm{P}$ (Twist-bcd) insertion was mapped to the second chromosome and balanced by $\mathrm{CyO}$ were individually crossed to $\mathrm{Sp} / \mathrm{CyO}$; $\mathrm{Toll}^{\mathrm{rm}}$ /TM3 females, which were isolated by crossing Toll ${ }^{\mathrm{rm} 9} / \mathrm{TM} 3$ males to double balancer Sp/CyO; PrDr/TM3 females. twist-bcd/CyO; Toll ${ }^{\mathrm{rm} 9} / \mathrm{TM} 3$ males were crossed to Toll ${ }^{\mathrm{rm} 10 /}$ TM3 females, and embryos from twist-bcd/+; Toll ${ }^{\mathrm{rm} 9} / \mathrm{Toll}^{\mathrm{rm} 10}$ females were collected from at least three individual lines for analysis by in situ hybridization.

\section{Whole-embryo extracts and western blotting}

Flies were allowed to deposit eggs on fresh apple juice agar plates with yeast paste for 2 hours, and then removed. Embryos were collected after aging an additional 2 hours at room temperature and homogenized in RIPA Buffer [50 mM TrisHCl (pH 8.0), $150 \mathrm{mM}$ $\mathrm{NaCl}, 1 \% \mathrm{NP}-40,0.5 \%$ deoxycholate, $0.1 \%$ SDS and protease inhibitors]. Extracts were centrifuged at $16,000 \mathrm{~g}$ for 15 minutes. The clear protein supernatant was carefully separated from floating lipid and precipitate, and quantified by Bradford assay. Equivalent amounts of embryo extracts were subjected to SDS-PAGE and western blotting using an affinity-purified, rabbit polyclonal antibody to Pelle protein at a 1:2000 dilution (kindly provided by James Manley) (Shen and Manley, 1998).

\section{RESULTS}

The snail, sim, vnd and sog expression patterns represent four different Toll-Dorsal signaling thresholds. snail is activated 


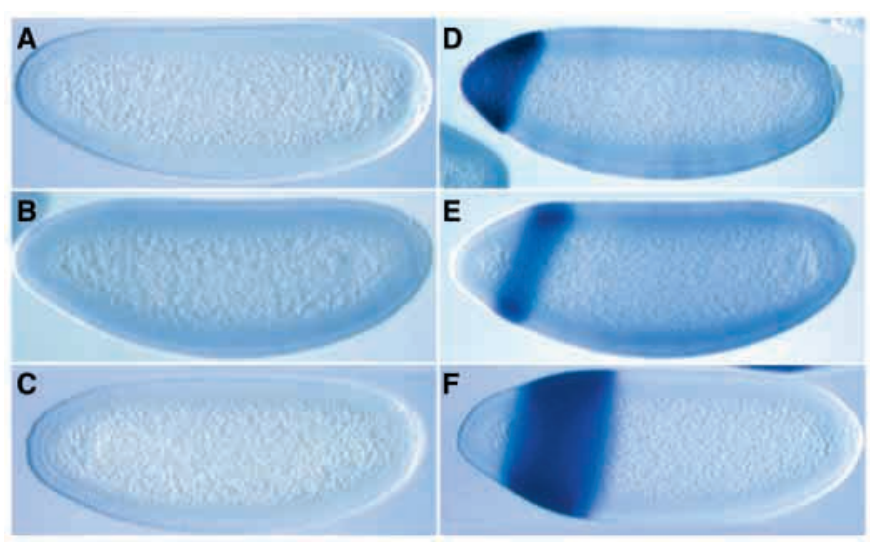

Fig. 1. sna, vnd and sog expression in transgenic embryos with an ectopic, anterior-posterior Dorsal nuclear gradient. Cellularizing mutant embryos were collected from females homozygous for a null mutation in the gastrulation defective $(g d)$ gene. Mutant embryos lack a dorsoventral Dorsal nuclear gradient. The embryos were hybridized with different digoxigenin-labeled antisense RNA probes, and visualized by histochemical staining. Each image is representative of the predominant pattern exhibited in the majority of embryos stained. In addition, at least three independent transgenic lines were analyzed for this and subsequent figures. Embryos are oriented with anterior to the left and dorsal up. (A-C) snail, vnd, and sog patterns, respectively, in mutant embryos. There is no staining above background levels. (D-F) snail, vnd, and sog patterns, respectively, in mutant embryos that contain the Toll ${ }^{10 b}$ transgene $\left[\mathrm{P}\left(\mathrm{bcdToll}{ }^{10} \mathrm{bcd}\right)\right]$. snail expression is detected at the anterior pole, a band of $v$ nd staining is detected in anterior regions (E), and a broad band of $\operatorname{sog}$ staining is detected in the anterior third of the embryo (F). The absence of $v n d$ and sog expression at the anterior pole is probably due to repression by Snail (D).

only by peak levels of the Dorsal gradient (Ip et al., 1992a), sim and vnd are activated by intermediate levels (Kasai et al., 1998; von Ohlen and Doe, 2000), and $\operatorname{sog}$ is activated by the lowest levels of the gradient (Francois et al., 1994). These expression patterns were visualized in mutant and transgenic embryos via in situ hybridization using digoxigenin-labeled antisense RNA probes (Huang et al., 1997).

\section{Activated Pelle generates multiple Toll-Dorsal patterning thresholds}

Dorsal target genes are essentially silent in mutant embryos that lack an endogenous dorsoventral Dorsal nuclear gradient (Fig. 1A-C). Mutant embryos were collected from females that are homozygous for a null mutation in the gastrulation defective $(g d)$ gene, which blocks the processing of the Spätzle ligand and the activation of the Toll receptor. These mutants permit the analysis of ectopic, anteroposterior Dorsal and Twist gradients in 'apolar' embryos that lack dorsoventral polarity. snail (Fig. 1D), vnd, (Fig. 1E) and sog (Fig. 1F) are sequentially expressed along the anteroposterior axis of mutant embryos that contain a constitutively activated form of the Toll receptor $\left(\mathrm{Toll}^{10 \mathrm{~b}}\right)$ misexpressed at the anterior pole using the bicoid $(b c d)$ promoter and 3' UTR (Fig. 1). These expression patterns depend on an ectopic anteroposterior Dorsal nuclear gradient (Huang et al., 1997). The repression of the vnd and sog patterns at the anterior pole is probably mediated by Snail, which normally excludes expression of these genes in the ventral mesoderm of wild-type embryos (Mellerick and Nirenberg, 1995; Rusch and Levine, 1996).

The activated Pelle-Tor ${ }^{4021}$ kinase also directs sequential anteroposterior patterns of snail, vnd, and sog expression in $g d^{-} / g d^{-}$mutant embryos (Fig. 2C-E). As in the case of Toll ${ }^{10 b}$, the activated Pelle kinase was misexpressed at the pole using the bcd 3' UTR. The snail, vnd and sog expression patterns are similar to those obtained with the Toll ${ }^{10 \mathrm{~b}}$ transgene (see Fig. 1D-F). The vnd and sog expression patterns are probably repressed at the anterior pole by Snail (Fig. 2D,E). These results suggest that the levels of Pelle kinase activity are sufficient to determine different Dorsal transcription thresholds.

Similar experiments were carried out with a Pelle-Tor fusion gene that contains the Tor signal peptide, extracellular domain and transmembrane peptide, but lacks the amino acid substitution (Y327C) in the Tor ${ }^{4021}$ protein that induces dimerization (Fig. 2F-H). The Pelle-Tor fusion protein fails to induce snail expression (Fig. 2F), but succeeds in activating vnd (Fig. 2G) and sog (Fig. 2H). The activities of the PelleTor ${ }^{4021}$ and Pelle-Tor proteins are summarized in Fig. 2B.

Western assays were carried out to determine whether the different activities of Pelle-Tor ${ }^{4021}$ and Pelle-Tor might result from the differential stability of the two fusion proteins (Fig. 2A). The normal Pelle kinase has a molecular weight of $\sim 75$ kDa (lane 1, Fig. 2A), whereas the Pelle-Tor ${ }^{4021}$ and Pelle-Tor fusion proteins are considerably larger, $140 \mathrm{kDa}$ (lanes 2 and 3 , respectively). The Pelle-Tor fusion protein (lane 3) is expressed at somewhat higher levels than the Pelle-Tor ${ }^{4021}$ protein, but nonetheless fails to activate snail expression. One interpretation of these results is that recruitment to the plasma membrane is not sufficient for the induction of peak Pelle kinase activity. Rather, full induction might require both recruitment and protein dimerization, which is achieved with the PelleTor ${ }^{4021}$ fusion protein, but not with Pelle-Tor (Grosshans et al., 1994; Galindo et al., 1995). The PelleTor ${ }^{4021}$ protein is constitutively activated in the absence of the Tor ligand, whereas full activation of Pelle-Tor might require ligand (see Discussion).

\section{Synergistic activities of Dorsal and Twist}

There are several similarities between Dorsal and the major determinant of anteroposterior patterning, Bicoid, even though the two morphogens are unrelated. Both gradients activate regulatory genes that are essential for patterning the early embryo. Bicoid activates the zinc finger gene Hunchback, while Dorsal activates the bHLH gene Twist. It has recently been shown that the loss of the normal Bicoid gradient can be largely compensated by an anteroposterior Hunchback gradient (Wimmer et al., 2000). Hunchback restores posterior head segments and the thoracic segments lost in bicoid-/bicoid $^{-}$ mutant embryos. We have investigated the possibility that Twist plays a similar role in dorsoventral patterning.

Dorsoventral patterning genes exhibit abnormal patterns of expression in twist ${ }^{-} /$twist $^{-}$mutant embryos (Fig. 3). For example, snail expression is reduced, and the residual snail pattern exhibits periodicity along the anteroposterior axis (Fig. 3C,D; compare with 3A,B). The activities of the constitutively activated Toll ${ }^{10 \mathrm{~b}}$ receptor are significantly impaired in twist ${ }^{-}$twist ${ }^{-}$mutant embryos (Fig. 3E,F). This experiment involved the use of an hsp83-Toll ${ }^{10 \mathrm{~b}}-3^{\prime}$ bcd UTR transgene, 


\section{A. Stathopoulos and M. Levine}

Fig. 2. Activated Pelle kinases generate multiple dorsoventral patterning thresholds. Mutant embryos at the cellular blastoderm stage were collected from females homozygous for a null mutation in $g d$. The embryos lack an endogenous dorsoventral Dorsal nuclear gradient. The embryos in C-E contain the PelleTor $^{4021}$ transgene, while the embryos in F-H contain a Pelle-Tor fusion gene. Both transgenes were expressed at the anterior pole of mutant embryos using the bcd 3' UTR. Embryos were stained with snail $(\mathrm{C}, \mathrm{F})$, vnd $(\mathrm{D}, \mathrm{G})$, and $\operatorname{sog}(\mathrm{E}, \mathrm{H})$ hybridization probes. (A) Total protein extracts from wild-type and transgenic embryos were fractionated on a polyacrylamide gel, transferred to a membrane, and then incubated with an anti-Pelle antiserum. Lane 1 contains an extract from wild-type embryos $(y w)$. The Pelle kinase has an apparent molecular weight of $\sim 75 \mathrm{kDa}$. Lanes 2 and 3 contain extracts from transgenic embryos carrying either the Pelle-Tor ${ }^{4021}$ (lane 2) or the Pelle-Tor (lane 3) fusion protein. Both full-length proteins have an apparent molecular weight of $\sim 140 \mathrm{kDa}$ (the band at $\sim 95 \mathrm{kDa}$ is a putative degradation product). There are slightly higher levels of Pelle-Tor (lane 3) than PelleTor $^{4021}$ (lane 2). (B) The diagram depicts a precellular embryo that contains either the Pelle-Tor 4021 or the Pelle-Tor transgene. Pelle-Tor ${ }^{4021}$ leads to the activation of snail at the anterior pole, whereas even high levels of the Pelle-Tor transgene fail to induce snail expression. (C) snail expression in mutant embryos that contain the Pelle-Tor ${ }^{4021}$ transgene. Staining is restricted to anterior regions containing peak levels of the transgene. (D) vnd expression pattern. Staining is detected in a stripe just posterior to the snail expression pattern. (E) sog expression pattern. Staining is detected in a broad band in the anterior third of the embryo. Both vnd and sog are repressed at the anterior pole, probably by Snail.(F) snail is not expressed in mutant embryos that contain the Pelle-Tor transgene. (G) vnd is activated only at the very anterior tip of embryos that express the Pelle-Tor transgene. $(\mathrm{H})$ sog exhibits a broader pattern of expression in these embryos.

which induces a broad anteroposterior Dorsal nuclear gradient (Huang et al., 1997). This transgene is similar to the one used in Fig. 1D-F, except that Toll ${ }^{10 b}$ was expressed using the stronger $h s p 83$ promoter rather than the bicoid promoter. In an otherwise normal genetic background, the Toll ${ }^{10 \mathrm{~b}}$ transgene leads to the ectopic activation of snail expression in the presumptive head and anterior thorax (Fig. 3E). However, in twist ${ }^{-} /$twist $^{-}$embryos there is reduced expression of both the endogenous snail expression pattern, and the ectopic pattern in anterior regions (Fig. 3F). Thus, even abnormally high levels of Toll signaling cannot compensate for the loss of Twist. The gap in snail expression seen at the boundary between the ectopic and endogenous patterns (arrow, Fig. 3E) may be due to an unknown repressor that is regulated by low levels of the Dorsal gradient. This gap is more pronounced in twist $/$ twist mutants (arrow, Fig. 3F), suggesting that Twist is not required for the activation of the putative snail repressor.

The preceding results suggest that Twist is necessary for specifying Dorsal gradient thresholds. To determine whether Twist might be sufficient, a twist-bcd transgene was introduced into a number of genetic backgrounds. In wild-type embryos, the twist-bcd transgene induces ectopic activation of the sim gene in anterior regions (Fig. 4D; compare with 4A). This activation is more easily visualized in snail ${ }^{-} /$snail $^{-}$mutant embryos because Snail represses sim expression in the ventral mesoderm (Kasai et al., 1992). There is a delay in the onset of

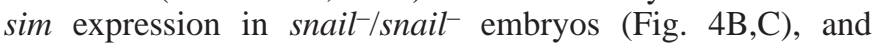
staining is sporadic in ventral regions where the gene is normally repressed (Fig. 4C; compare with 4A). However, the twist-bcd transgene, when introduced into snail-/snail ${ }^{-}$mutant
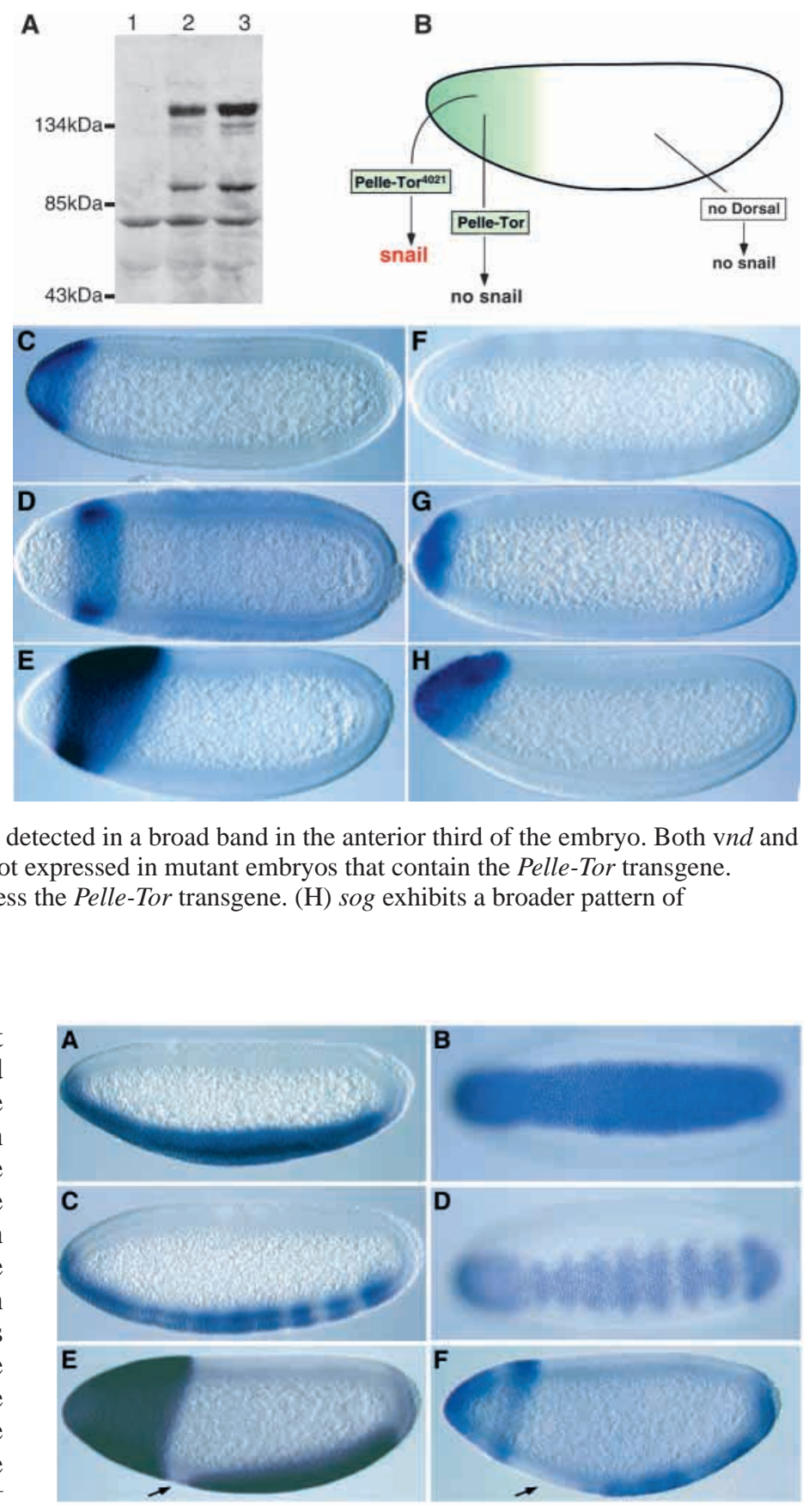

Fig. 3. Twist is required for Dorsal gradient thresholds. Embryos were hybridized with a digoxigenin-labeled snail antisense RNA probe. They are oriented with anterior to the left. $(A, B)$ Lateral $(A)$ and ventral (B) views of wild-type embryos. snail staining is restricted to the ventral mesoderm. (C,D) Lateral (C) and ventral (D) views of twist/twist mutant embryos. The snail staining pattern is diminished and exhibits periodic repression along the anterior-posterior axis. (E,F) Wild-type (E) and mutant embryos (F) expressing a Toll ${ }^{10 b}$ transgene $\left[\mathrm{P}\left(\mathrm{hspToll}{ }^{10 \mathrm{~b}} \mathrm{bcd}\right)\right]$, which produces a broad ectopic Dorsal nuclear gradient along the anterior-posterior axis. In a wild-type background the transgene produces uniformly intense activation of snail in the anterior third of the embryo, in addition to the endogenous pattern in the ventral mesoderm (E). There is a gap in staining where the ectopic and endogenous snail patterns intersect (arrow). There is a severe reduction in both the endogenous and ectopic snail expression patterns in twist $^{-} /$twist $^{-}$mutant embryos (F). 
Fig. 4. An anterior-posterior Twist gradient induces ectopic expression of sim. Embryos were hybridized with a digoxigenin-labeled $\operatorname{sim}(\mathrm{A}-\mathrm{F})$ or Sex-lethal (G-I) antisense RNA probe and are oriented with anterior to the left. $(\mathrm{A}, \mathrm{D})$ Wild-type embryos that lack (A) or express (D) a twistbcd transgene. sim is normally expressed in two lines that straddle the presumptive mesoderm (A). Staining does not extend to the anterior pole. In contrast, the twist$b c d$ transgene induces ectopic expression of sim at the anterior pole (D). This staining is first detected in precellular embryos (not shown), and persists during cellularization (D) and gastrulation (not shown). (B,C) snail $^{-} /$snail $^{-}$mutant embryos that lack the $t w i s t-b c d$ transgene. There is a delay in the onset of expression, and staining is not detected in advanced cellularizing embryos (B). Sporadic and weak expression is first detected at the onset of gastrulation in ventral regions (C). B and C display lateral and ventral views, respectively. (E,F) snail ${ }^{-} /$snail $^{-}$mutant embryos that express the twist-bcd transgene. There is strong activation of sim in anterior regions of cellularizing embryos (E and F display lateral and ventral reviews, respectively). Strong ectopic expression persists during germband elongation (not shown). (G-I) Sex-lethal expression in wild-type embryos and those expressing the twist-bcd transgene. Sex-lethal is ubiquitously expressed in female embryos $(\mathrm{H})$ and is not expressed in male embryos $(\mathrm{G})$. In embryos from females containing the twist-bcd transgene, Sexlethal is repressed at the anterior end of the embryo (I) in the domain that coincides with ectopic Twist expression. All Sex-lethal expressing embryos exhibit this repression, which accounts for the daughterless phenotype exhibited by females containing the $t w i s t-b c d$ transgene.

embryos, induces strong expression of $\operatorname{sim}$ in anterior regions (Fig. 4E,F), although staining is largely restricted to ventral regions where there are high levels of the Dorsal gradient (Fig. 4F).

In addition, females containing the twist-bcd transgene exhibit a daughterless phenotype as only male progeny are observed. We examined Sex-lethal expression in embryos obtained from these females. Sex-lethal is required for sex determination in females, and this gene is expressed in female but not male embryos (Fig. 4H,G, respectively) (Bopp et al., 1991). Sex-lethal expression is repressed in the anterior of embryos obtained from females containing the twist-bcd transgene (Fig. 4I). This result suggests that Twist may form heterodimeric complexes with Daughterless/Scute, two bHLH proteins required for Sex-lethal expression, thereby titrating the levels of these proteins and making them unable to activate Sex-lethal. The ability of Twist to form homodimeric as well as heterodimeric complexes with other bHLH proteins present in the early embryo may regulate its own dorsoventral patterning activities as well (see Discussion).

\section{A Twist gradient can generate multiple dorsoventral patterning thresholds}

In order to determine whether Twist patterning activities require a Dorsal gradient, the twist-bcd transgene was introduced into mutant embryos derived from Toll $^{\mathrm{rm} 9} /$ Toll $^{\mathrm{rm} 10}$ transheterozygous females. This mutant Toll receptor is defective and partially active in a Spätzle-independent fashion (Schneider et al., 1991). Consequently, mutant embryos contain uniform, low levels of the Dorsal protein in both dorsal and ventral nuclei. The twist-bcd transgene causes a substantial reorganization in the patterning of mutant embryos (Fig. 5).

The low, uniform levels of Dorsal present in Toll $^{\text {rm9 }} /$ Toll $^{\text {rm10 }}$ mutant embryos are insufficient to activate snail in precellular or newly cellularized embryos (Fig. 5A). The twist-bcd transgene activates snail throughout anterior regions of precellular embryos (Fig. 5B); however, this staining pattern is refined into a broad anterior stripe after cellularization (Fig. 5C). This ectopic snail expression pattern exhibits a sharp

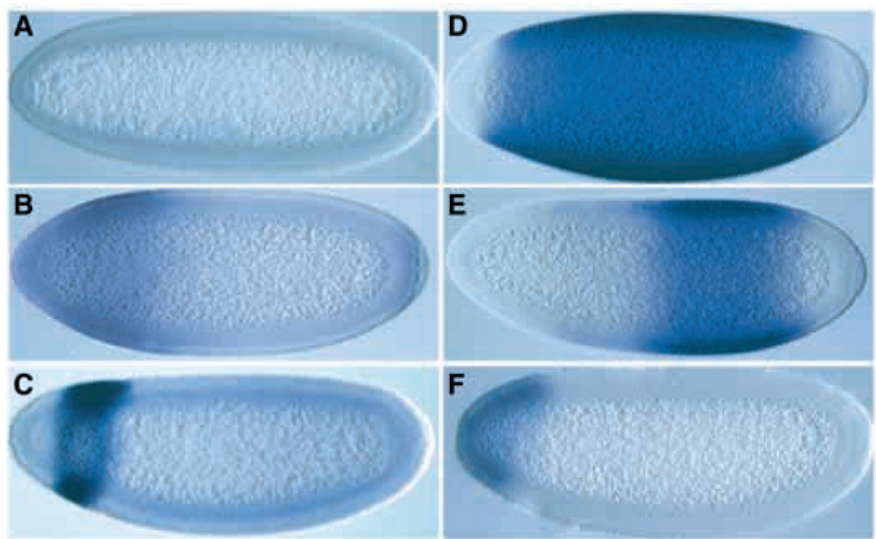

Fig. 5. An anterior-posterior Twist gradient specifies two dorsoventral patterning thresholds in the absence of a Dorsal gradient. Embryos were hybridized with a digoxigenin-labeled snail antisense RNA probe, and are oriented with anterior to the left and dorsal up. (A) snail is not expressed in mutant embryos derived from Toll $^{r m 9} /$ Toll $^{r m 10}$ transheterozygous females. sim is also silent in these mutants (data not shown). (B) snail staining pattern in a mutant embryo that contain the twist-bcd transgene. snail is broadly activated in anterior regions of cellularizing embryos (compare with A). (C) The snail staining pattern sharpens in cellularized mutant embryos that express the twist-bcd transgene. Staining is lost at the anterior pole. (D) sog is expressed throughout Toll $^{r m 9} /$ Toll $^{r m 10}$ mutant, with the exception of the termini. (E) sog staining pattern in mutant embryos that contain the twist-bcd transgene. Staining is repressed in anterior regions (compare with D) where the Snail repressor is ectopically activated (see B). (F) sim staining pattern in mutant embryos that express the twist-bcd transgene. Expression is restricted to the anterior pole, in regions where the Snail repressor is lost (C). 


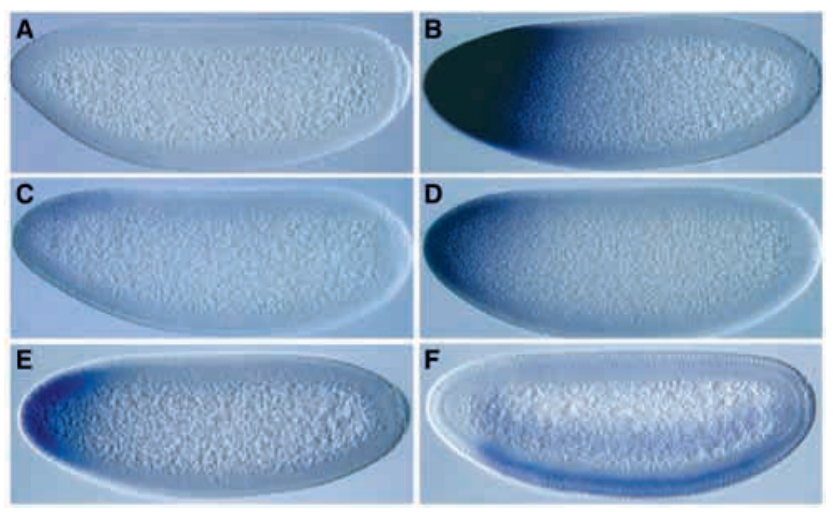

Fig. 6. Twist can activate $v n d$ and sim without Dorsal. Embryos were stained with twist (A,B), sim (C), and $v n d(\mathrm{D}-\mathrm{F}) \mathrm{RNA}$ probes, and are oriented with anterior to the left and dorsal up. (A,B) Mutant embryos were obtained from $g d^{-} / g d^{-}$females and completely lack Dorsal nuclear protein. There is no twist expression in these mutants (A), but twist exhibits intense expression in anterior regions of mutant embryos that contain the twist-bcd transgene (B). (C,D) sim (C) and $v n d$ (D) staining patterns in $g d^{-} / g d^{-}$mutant embryos that contain the twist-bcd transgene. Staining is detected in anterior regions where there are high levels of Twist. (E,F) The vnd expression pattern in precellular (E) and cellularized (F) wild-type embryos that contain the twist-bcd transgene. $v$ nd expression is induced at the anterior pole in early embryos by the $t$ wist-bcd transgene (E), but this anterior expression is lost during the onset of the endogenous expression pattern (ventral stripes) after cellularization $(\mathrm{F})$.

posterior border, suggesting that the Twist gradient is sufficient for the on/off regulation of snail in the absence of a Dorsal gradient (see Discussion).

sog is normally activated throughout the neurogenic ectoderm by the lowest levels of the Dorsal gradient (Francois et al., 1994; Markstein et al., 2002). The low levels of Dorsal present in Toll $^{r m 9} /$ Toll $^{\text {rm10 }}$ mutant embryos are sufficient to activate sog everywhere except the extreme termini (Fig. 5D). The twist-bcd transgene leads to the loss of sog expression in anterior regions (Fig. 5E), probably because of repression by Snail. As mentioned earlier, Snail also appears to repress vnd and sog expression in anterior regions of transgenic embryos that contain the Toll $^{10 b}$ or Pelle-Tor ${ }^{4021}$ transgenes (see Figs 1 and 2).

The low levels of Dorsal present in Toll $^{r m 9} /$ Toll $^{r m 10}$ mutant embryos are insufficient to activate sim, although there is occasional staining in the posterior pole (data not shown). The twist-bcd transgene leads to the efficient activation of sim in anterior regions (Fig. 5F). Staining appears to be restricted to those regions where snail expression is lost (Fig. 5C). These results suggest that a Twist gradient is sufficient to generate multiple dorsoventral patterning thresholds (sim and snail) in the presence of low, uniform levels of Dorsal.

The twist-bcd transgene was introduced into mutant embryos that completely lack Dorsal (Fig. 6). Without the transgene these mutants do not express twist, snail, sim, vnd or sog (Fig. 6A; data not shown). Introduction of the twist-bcd transgene causes intense expression of twist in the anterior $40 \%$ of the embryo (Fig. 6B; compare with 6A). This broad Twist gradient fails to activate snail (data not shown), but succeeds in inducing weak expression of $\operatorname{sim}$ (Fig. 6C) and somewhat stronger staining of vnd (Fig. 6D) at the anterior pole. The activation of $v n d$ in mutant embryos is comparable with the expression seen in wild-type and Toll $^{r m 9} /$ Toll $^{\text {rm10 }}$ embryos (Fig. $6 \mathrm{E}$; data not shown). However, in both wild-type and mutant embryos the vnd pattern is transient, and lost after the completion of cellularization (Fig. 6F; data not shown). These results indicate that Twist can activate dorsoventral patterning genes in the absence of Dorsal.

\section{DISCUSSION}

\section{Linear signaling}

The demonstration that the Pelle-Tor ${ }^{4021}$ fusion protein can generate most or all dorsoventral patterning thresholds suggests that the levels of Pelle kinase activity determine Spätzle-Toll signaling thresholds. There is no need to invoke branching in the Toll pathway upstream of Pelle, although it is possible that branching exists downstream of Pelle. For example, there is emerging evidence that IkB kinases might trigger the degradation of Cactus in the immune response (Rutshmann et al., 2000; Silverman et al., 2000; Lu et al., 2001). It is currently unclear whether these kinases are also required for dorsoventral patterning and, if so, whether they are induced by Pelle or separately activated in response to Toll signaling.

Multiple patterning thresholds are also specified by different levels of kinase activity in the Tor, epidermal growth factor receptor (EGFR) and Sevenless (Sev) signaling pathways (Greenwood and Struhl, 1997; Halfar et al., 2001). In the embryo, low levels of Ras1 are sufficient to activate the Tor target gene tailless, whereas higher levels are required for the induction of hückebein (Greenwood and Struhl, 1997). Similarly, in the eye, different levels of EGFR and Sev signaling lead to different levels of MAPK activity: low levels permit the differentiation of R8 photoreceptor cells, whereas higher levels (or more persistent expression) of MAPK activity leads to the specification of an R1-R7 fate (Halfar et al., 2001). In the present study it has been possible to link the levels of an activated cytoplasmic kinase (Pelle) with the expression of well-characterized target genes that are regulated by different concentrations of the Dorsal gradient. It is conceivable that the MAP and Pelle kinases exist in multiple states, which help establish different thresholds of gene activity. For example, even high concentrations of membrane-localized PelleTor fusion protein fail to activate snail. While the Tor ${ }^{4021}$ receptor domain of Pelle-Tor ${ }^{4021}$ induces ligand-independent dimerization, the wild-type Tor receptor domain contained within the Pelle-Tor fusion might mediate only low levels of dimerization because of competition with the endogenous Tor receptor for binding to the Trunk ligand (Casali and Casanova, 2001). Full activation of the Pelle kinase might depend on recruitment to the plasma membrane and dimerization (Grosshans et al., 1994; Galindo et al., 1995), while recruitment to the membrane alone may produce only partial activation of kinase activity. The induction of full Pelle kinase activity, and ultimately the activation of snail expression, might require trans-phosphorylation induced by dimerization of the Toll receptor (Shen and Manley, 1998). In the context of the normal Toll-Dorsal signaling pathway, the transition between a partially activated Pelle kinase and a fully activated kinase 
Fig. 7. Dorsal-Twist patterning activities. (A) Previous studies identified a cluster of three low affinity Dorsal operator sites in a distal region of the snail 5' cis-regulatory region. The proximal regulatory region contains two Twist binding sites. The diagrams above the representation of the snail gene depict cross sections of embryos with Dorsal (left) and Twist (right) protein gradients (there are peak levels in ventral regions). The diagram to the far right summarizes a mutant embryo derived from Toll $^{\text {rm } 9} /$ Toll $^{\text {rm10 }}$ females that contains a twist-bcd transgene. Normally, these embryos lack snail expression, but the twist-bcd transgene leads to the activation of snail in anterior regions. These results suggest that Twist plays a critical role in the on/off regulation of snail expression since the mutant embryos lack a Dorsal gradient. (B) Summary of Dorsal/Twistregulated expression of snail and sim. We find that high amounts of Twist-bcd support ectopic sim expression (blue) that requires at least low levels of nuclear Dorsal and is repressed by Snail. High levels of ectopic nuclear Dorsal supported by the Toll ${ }^{10 b_{-}}-b c d$ transgene produces only very low levels of snail expression (red) and no sim expression (data not shown) in the absence of Twist. We conclude that Dorsal and Twist transcription factors function interdependently in order to generate multiple thresholds of gene expression. (sna-: no Snail protein; $g d$-: no nuclear Dorsal protein; $t w i^{-}$: no Twist protein; Toll ${ }^{r m 9 / r m 10}$ : low levels of nuclear Dorsal protein).

might help generate a sharp on/off pattern of snail expression. Perhaps the Pelle kinase is converted into the fully activated form (required for snail expression) when Toll signaling exceeds a certain minimal threshold.

\section{Twist gradient thresholds}

An anteroposterior Twist gradient generates at least two thresholds of gene activity in mutant embryos that contain decreased levels of Dorsal (summarized in Fig. 7B). High levels of Twist activate $\operatorname{sim}$ at the anterior pole, whereas lower levels are sufficient to induce the expression of snail in more posterior regions of embryos containing low, uniform levels of the Dorsal protein (Fig. 5C,F). These results demonstrate that twist gene activity is not dedicated to mesoderm formation. Instead, Twist supports expression of two regulatory genes, sim and vnd, which pattern ventral regions of the neurogenic ectoderm (Crews et al., 1988; McDonald et al., 1998). The twist-bcd transgene was shown to induce weak expression of both genes even in mutant embryos that completely lack Dorsal (Fig. 6C,D).

The Twist gradient exhibits some unexpected activities in Toll $^{\text {rm9 }} /$ Toll $^{\text {rm10 }}$ mutant embryos. In particular, both high and intermediate levels of the gradient initially activate snail in a broad domain of precellular embryos (Fig. 5B). However, during cellularization snail is repressed at the anterior pole where there are high levels of Twist (Fig. 5C). Thus, it would appear that high levels of Dorsal + high levels of Twist activate snail expression (e.g. ventral region of wild-type embryos), while low levels of Dorsal + high levels of Twist repress expression (e.g. anterior region of Toll $^{\mathrm{rm} 9} / \mathrm{Toll}^{\mathrm{rm} 10}$ embryos containing the twist-bcd transgene). The ratio of Dorsal to Twist might keep Twist patterning activity under control, such that high levels of Twist specify mesodermal targets (i.e. snail), lower levels specify neurogenic targets (i.e. sim and vnd) and the expression of genes such as Sxl remain unaffected.

Alternatively, Twist may differentially interact with a number of bHLH proteins that are present in the early embryo (Moore et al., 2000) to affect its patterning activity. For example, Twist is thought to form a heteromeric activation complex with other bHLH proteins including the ubiquitous, maternal bHLH protein Daughterless (Da) (Gonzalez-Crespo and Levine, 1993; Castanon et al., 2001). The loss of Sex-lethal expression at the anterior pole of twist-bcd embryos (Fig. 4I) may result from a failure of Twist-Daughterless heterodimers to activate this gene. It has been demonstrated that Twist-Daughterless heterodimers possess a different patterning activity from Twist-Twist homodimers (Castonon et al., 2001). It is possible that TwistDaughterless heterodimers formed in twist-bcd embryos actively repress Sex-lethal expression. Alternatively, Twist might titrate Daughterless levels by forming a sterile heterodimeric complex that is not able to activate Sex-lethal. However, Sex-lethal is normally expressed in ventral regions of wild-type embryos that contain both Twist and the ubiquitous Daughterless, therefore regulation of bHLH patterning activities 
must be more complex. In relation to dorsovental patterning, Twist-Twist complexes might be favored in anterior regions of embryos that express the twist-bcd transgene, while TwistbHLH complexes are formed away from the anterior pole where expression of the transgene is decreased. These complexes might fail to activate snail, or even actively repress transcription as it has been demonstrated that several bHLH proteins can function as repressors of transcription (Brentrup et al., 2000). Regardless of mechanism, the Twist gradient inverts the order of the sequentially expressed snail and sim genes, when compared with the patterns obtained with the normal Dorsal (and Twist) gradient. In the presence of low, uniform levels of Dorsal, high concentrations of Twist specify mesectoderm ( $\mathrm{sim}$ expression), while lower levels specify mesoderm (snail expression). These observations raise the possibility of evolutionary plasticity in the use of Twist in tissue specification.

\section{Dorsal-Twist synergy}

snail is activated by Dorsal and Twist in cellularizing embryos (Ip et al., 1992a). The sharp lateral limits of the snail expression pattern establish the boundary between the presumptive mesoderm and neurogenic ectoderm (Kosman et al., 1991; Leptin, 1991). It has been suggested that the crude Dorsal gradient triggers a somewhat steeper Twist gradient, and the two activators function synergistically within the snail 5' cis-regulatory DNA to establish the sharp, on/off expression pattern (Ip et al., 1992a). Dorsal-Twist transcription synergy may provide a means for 'multiplying' the Dorsal and Twist gradients to produce the sharp snail pattern (see Summary, Fig. 7A). This model suggests that both proteins must be present in a gradient to generate the sharp snail border. However, while both Dorsal and Twist are required for the activation of snail, we have shown that a Twist gradient is sufficient to generate a reasonably sharp pattern of snail expression in embryos containing low, uniform levels of Dorsal. We propose that cooperative binding of Twist might act as a switch to regulate snail expression when the snail $5^{\prime}$ cis-regulatory region is rendered responsive by the Dorsal activator (whether present at uniform levels or in a gradient). Therefore, the ratio of Dorsal to Twist may be important to produce the sharp lateral limits of snail expression.

This study raises some questions about the role of operator binding affinities in the specification of different transcription thresholds. The Dorsal binding sites present in the snail 5' regulatory region bind with lower affinity than the sites present in the rho lateral stripe enhancer (NEE) (Ip et al., 1992a; Ip et al., 1992b). The analysis of a number of synthetic enhancers prompted the proposal that the activation of Dorsal target genes in the ventral mesoderm versus lateral neurogenic ectoderm depends on the affinity of Dorsal operator sites (Jiang and Levine, 1993; Huang et al., 1997). However, the demonstration that the twist-bcd transgene can activate snail expression in Toll $^{r m 9} /$ Toll $^{r m 10}$ embryos suggests that occupancy of the distal Dorsal-binding sites may not be crucial for determining whether the gene is on or off. It is conceivable that Dorsal occupies one or more sites in mutant embryos, but is unable to trigger expression in the absence of Twist. In general, 'promoter context' (combinations of regulatory factors) might be more critical for defining Dorsal transcription thresholds than the affinities of Dorsal operator sites.

The relationship between Dorsal and Twist appears distinct from the interplay between Bicoid and Hunchback (Wimmer et al., 2000). It has been suggested that the Bicoid gradient is a relatively recent evolutionary innovation for patterning the anterior-posterior axis of long germband insects (Dearden and Akam, 1999). By contrast, Hunchback is ancient and is used in the patterning of short germband insects such as grasshoppers. Most of the patterning activity controlled by the Bicoid gradient appears to be mediated by Hunchback, which is a direct target of the Bicoid activator. Only the patterning of the anteriormost head structures requires Bicoid and cannot be compensated by high levels of Hunchback (Wimmer et al., 2000). Thus, the patterning activity of the Bicoid gradient can be explained by the regulation of several target genes, which is consistent with its recent evolution. By contrast, either Dorsal or Twist protein alone produces only a small subset of the five or six dorsoventral patterning thresholds generated by the concerted action of both proteins. We conclude that Dorsal and Twist work in a highly interdependent and synergistic fashion to regulate a large number of target genes involved in patterning the dorsoventral axis.

We thank Tony Ip for providing snail mutant flies, Maria Leptin for sending a full-length twist cDNA plasmid, James Manley for providing the anti-Pelle antibody, and Steve Wasserman for sending Pelle-Tor and Pelle-Tor ${ }^{4021}$ plasmids. This work was funded by a grant from the NIH (GM46638) A. S. is supported by a postdoctoral fellowship from the NIH (GM20352).

\section{REFERENCES}

Baylies, M. K. and Bate, M. (1996). twist: a myogenic switch in Drosophila. Science 272, 1481-1484.

Belvin, M. P. and Anderson, K. V. (1996). A conserved signaling pathway: the Drosophila toll-dorsal pathway. Annu. Rev. Cell Dev. Biol. 12, 393-416.

Bopp, D., Bell, L. R., Cline, T. W. and Schedl, P. (1991). Developmental distribution of female-specific Sex-lethal proteins in Drosophila melanogaster. Genes Dev. 5, 403-415.

Brentrup, D., Lerch, H.-P., Jackle, H. and Noll, M. (2000). Regulation of Drosophila wing vein patterning: net encodes a bHLH protein repressing rhomboid and is repressed by rhomboid-dependent Egfr signalling. Development 127, 4729-4741.

Casali, A. and Casanova, J. (2001). The spatial control of Torso RTK activation: a C-terminal fragment of the Trunk protein acts as a signal for Torso receptor in the Drosophila embryo. Development 128, 17091715.

Castanon, I., von Stetina, S., Kass, J. and Baylies, M. K. (2001). Dimerization partners determine the activity of the Twist bHLH protein during Drosophila mesoderm development. (2001). Development 128, 3145-3159.

Chen, G., Handel, K. and Roth, S. (2000). The maternal NF-kappaB/dorsal gradient of Tribolium castaneum: dynamics of early dorsoventral patterning in a short-germ beetle. Development 127, 5145-5156.

Crews, S. T., Thomas, J. B. and Goodman, C. S. (1988). The Drosophila single-minded gene encodes a nuclear protein with sequence similarity to the per gene product. Cell 52, 143-151.

Dearden, P. and Akam, M. (1999). Developmental evolution: Axial patterning in insects. Curr. Biol. 9, R591-R594.

Ferguson, E. L. (1996). Conservation of dorsal-ventral patterning in arthropods and chordates. Curr. Opin. Genet. Dev. 6, 424-431.

Francois, V., Solloway, M., O’Neill, J. W., Emery, J. and Bier, E. (1994). Dorsal-ventral patterning of the Drosophila embryo depends on a putative negative growth factor encoded by the short gastrulation gene. Genes Dev. 8, 2602-2616.

Galindo, R. L., Edwards, D. N., Gillespie, S. K. and Wasserman, S. A. (1995). Interaction of the pelle kinase with the membrane-associated protein tube is required for transduction of the dorsoventral signal in Drosophila embryos. Development 121, 2209-2218.

Gonzalez-Crespo, S. and Levine, M. (1993). Interactions between dorsal and 
helix-loop-helix proteins initiate the differentiation of the embryonic mesoderm and neuroectoderm in Drosophila. Genes Dev. 7, 1703-1713.

Greenwood, S. and Struhl, G. (1997). Different levels of Ras activity can specify distinct transcriptional and morphological consequences in early Drosophila embryos. Development 124, 4879-4886.

Grosshans, J., Bergmann, A., Haffter, P. and Nusslein-Volhard, C. (1994). Activation of the kinase Pelle by Tube in the dorsoventral signal transduction pathway of Drosophila embryo. Nature 372, 563-566.

Halfar, K., Rommel, C., Stocker, H. and Hafen, E. (2001). Ras controls growth, survival and differentiation in the Drosophila eye by different thresholds of MAP kinase activity. Development 128, 1687-1696.

Harfe, B. D., Vaz Gomes, A., Kenyon, C., Liu, J., Krause, M. and Fire, A. (1998). Analysis of a Caenorhabditis elegans Twist homolog identifies conserved and divergent aspects of mesodermal patterning. Genes Dev. 12, 2623-2635.

Huang, A. M., Rusch, J. and Levine, M. (1997). An anteroposterior Dorsal gradient in the Drosophila embryo. Genes Dev. 11, 1963-1973.

Ip, Y. T., Park, R. E., Kosman, D., Yazdanbakhsh, K. and Levine, M. (1992a). dorsal-twist interactions establish snail expression in the presumptive mesoderm of the Drosophila embryo. Genes Dev. 6, 15181530.

Ip, Y. T., Park, R. E., Kosman, D., Bier, E. and Levine, M. (1992b). The dorsal gradient morphogen regulates stripes of rhomboid expression in the presumptive neuroectoderm of the Drosophila embryo. Genes Dev. 6, 17281739.

Jiang, J., Kosman, D., Ip, Y. T. and Levine, M. (1991). The dorsal morphogen gradient regulates the mesoderm determinant twist in early Drosophila embryos. Genes Dev. 5, 1881-1891.

Jiang, J. and Levine, M. (1993). Binding affinities and cooperative interactions with bHLH activators delimit threshold responses to the dorsal gradient morphogen. Cell 72, 741-752.

Karin, M. (1999). The beginning of the end: IkappaB kinase (IKK) and NFkappaB activation. J Biol. Chem. 274, 27339-27342.

Kasai, Y., Nambu, J. R., Lieberman, P. M. and Crews, S. T. (1992). Dorsalventral patterning in Drosophila: DNA binding of snail protein to the singleminded gene. Proc. Natl. Acad. Sci. USA 89, 3414-3418.

Kasai, Y., Stahl, S. and Crews, S. (1998). Specification of the Drosophila CNS midline cell lineage: direct control of single-minded transcription by dorsal/ventral patterning genes. Gene Exp. 7, 171-189.

Konrad, K. D., Goralski, T. J. and Mahowald, A. P. (1988). Developmental genetics of the gastrulation defective locus in Drosophila melanogaster. Dev. Biol. 127, 133-142.

Kosman, D., Ip, Y. T., Levine, M. and Arora, K. (1991). Establishment of the mesoderm-neuroectoderm boundary in the Drosophila embryo. Science 254, 118-122.

Leptin, M. (1991). twist and snail as positive and negative regulators during Drosophila mesoderm development. Genes Dev. 5, 1568-1576.

Lu, Y., Wu, L. P. and Anderson, K. V. (2001). The antibacterial arm of the Drosophila innate immune response requires an IkappaB kinase. Genes Dev. 15, 104-110.

Markstein, M., Markstein, P., Markstein, V. and Levine, M. S. (2002). Genome-wide analysis of clustered Dorsal binding sites identifies putative target genes in the Drosophila embryo. Proc. Natl. Acad. Sci. USA 99, $763-$ 768

McDonald, J. A., Holbrook, S., Isshiki, T., Weiss, J., Doe, C. Q. and Mellerick, D. M. (1998). Dorsoventral patterning in the Drosophila central nervous system: the vnd homeobox gene specifies ventral column identity. Genes Dev. 12, 3603-3612.

Mellerick, D. M. and Nirenberg, M. (1995). Dorsal-ventral patterning genes restrict NK-2 homeobox gene expression to the ventral half of the central nervous system of Drosophila embryos. Dev. Biol. 171, 306-316.

Mizuguchi, K., Parker, J. S., Blundell, T. L. and Gay, N. J. (1998). Getting knotted: a model for the structure and activation of Spatzle. Trends Biochem. Sci. 23, 239-242.

Moore, A. W., Barbel, S., Jan, L. Y. and Jan, Y. N. (2000). A genomewide survey of basic helix-loop-helix factors in Drosophila. Proc. Natl. Acad. Sci. USA 97, 10436-10441.

Reuter, R. and Leptin, M. (1994). Interacting functions of snail, twist and huckebein during the early development of germ layers in Drosophila. Development 120, 1137-1150.

Rusch, J. and Levine, M. (1996). Threshold responses to the dorsal regulatory gradient and the subdivision of primary tissue territories in the Drosophila embryo. Curr. Opin. Genet. Dev. 6, 416-423.

Rutschmann, S., Jung, A. C., Zhou, R., Silverman, N., Hoffmann, J. A. and Ferrandon, D. (2000). Role of Drosophila IKK gamma in a tollindependent antibacterial immune response. Nat. Immunol. 1, 342-347.

Schneider, D. S., Hudson, K. L., Lin, T. Y. and Anderson, K. V. (1991). Dominant and recessive mutations define functional domains of Toll, a transmembrane protein required for dorsal-ventral polarity in the Drosophila embryo. Genes Dev. 5, 797-807.

Sen, J., Goltz, J. S., Stevens, L. and Stein, D. (1998). Spatially restricted expression of pipe in the Drosophila egg chamber defines embryonic dorsalventral polarity. Cell 95, 471-481.

Shen, B. and Manley, J. L. (1998). Phosphorylation modulates direct interactions between the Toll receptor, Pelle kinase and Tube. Development 125, 4719-4728.

Silverman, N., Zhou, R., Stoven, S., Pandey, N., Hultmark, D. and Maniatis, T. (2000). A Drosophila IkappaB kinase complex required for Relish cleavage and antibacterial immunity. Genes Dev. 14, 2461-2471.

Sprenger, F. and Nusslein-Volhard, C. (1992). Torso receptor activity is regulated by a diffusible ligand produced at the extracellular terminal regions of the Drosophila egg. Cell 71, 987-1001.

Thisse, C., Perrin-Schmitt, F., Stoetzel, C. and Thisse, B. (1991). Sequencespecific transactivation of the Drosophila twist gene by the dorsal gene product. Cell 65, 1191-1201.

Towb, P., Galindo, R. L. and Wasserman, S. A. (1998). Recruitment of Tube and Pelle to signaling sites at the surface of the Drosophila embryo. Development 125, 2443-2450.

von Ohlen, T. and Doe, C. Q. (2000). Convergence of dorsal, dpp, and egfr signaling pathways subdivides the drosophila neuroectoderm into three dorsal-ventral columns. Dev. Biol. 224, 362-372

Wimmer, E. A., Carleton, A., Harjes, P., Turner, T. and Desplan, C. (2000). Bicoid-independent formation of thoracic segments in Drosophila. Science 287, 2476-2479. 